\title{
Cost-Effectiveness Analysis of Amino Acid PET-Guided Surgery for Supratentorial High-Grade Gliomas
}

\author{
Alexander Heinzel ${ }^{1}$, Stephanie Stock ${ }^{2}$, Karl-Josef Langen ${ }^{3}$, and Dirk Müller ${ }^{2}$ \\ ${ }^{I}$ Department of Nuclear Medicine of the Heinrich-Heine University of Duesseldorf at the Research Centre Juelich, Juelich, Germany; \\ ${ }^{2}$ Institute for Health Economics and Clinical Epidemiology, University Hospital of Cologne, Cologne, Germany; and ${ }^{3}$ Institute for \\ Neuroscience and Medicine 4, Research Centre Juelich, Juelich, Germany
}

\begin{abstract}
High-grade gliomas are brain tumors associated with a devastating prognosis. Recent studies have indicated that the combined use of amino acid PET and MRI is superior to MRI alone to plan the surgical resection of high-grade gliomas. The aim of the study was to analyze the cost-effectiveness of the use of amino acid PET for the surgical resection of high-grade gliomas, compared with MRI alone, from the perspective of the national health insurance in Germany. Methods: A decision-tree model was set up to compare 2 strategies: the use of MRI alone and the combined use of MRI and PET for surgical resection of high-grade gliomas. For the analysis, 2 scenarios were calculated: a baseline scenario and a more expensive scenario, accounting for disease severity. To test the robustness of the results, probabilistic sensitivity analyses using Monte Carlo simulation were calculated. Results: Compared with MRI alone, the combined use of MRI and PET showed an incremental cost-effectiveness ratio of $€ 2,948$ $(€ 1 \sim$ U.S.\$1.3)per life-year gained for the baseline scenario and an incremental cost-effectiveness ratio of $€ 4,105$ per life-year gained for the admissible-charge-rate scenario. In the probabilistic sensitivity analysis in about $60 \%$ of the iterations, the combined use of PET and MRI was superior to MRI alone when assuming a willingness-to-pay threshold of $€ 30,000$. Conclusion: The model indicates that the combined use of MRI and PET may be cost-effective. The results of this analysis have to be considered carefully because there was only limited empiric evidence for several input parameters.
\end{abstract}

Key Words: amino acid PET; cost-effectiveness analysis; therapy planning; high-grade gliomas; PET guided surgery

J Nucl Med 2012; 53:552-558

DOI: 10.2967/jnumed.111.097352

H igh-grade gliomas are primary brain cancers that are difficult to treat because of their aggressive biology. They often result in massive neurologic damage, with a significant reduction in health-related quality of life $(1,2)$ and reduced life expectancy (3-5).

\footnotetext{
Received Sep. 7, 2011; revision accepted Dec. 1, 2011.

For correspondence or reprints contact: Alexander Heinzel, Department of Nuclear Medicine of the Heinrich-Heine University of Duesseldorf at the Research Centre Juelich, 52426 Juelich, Germany.

E-mail: a.heinzel@fz-juelich.de

Published online Mar. 14, 2012.

COPYRIGHT @ 2012 by the Society of Nuclear Medicine, Inc.
}

The surgical resection of the tumor, which is the initial treatment option (6), aims to remove as much cancer tissue as possible while preserving as much unaffected tissue as possible. To distinguish tumor and unaffected brain tissue, valid and reliable diagnostic methods are needed. MRI is considered the gold standard in diagnostic imaging of high-grade gliomas (7). However, MRI is limited in its ability to distinguish tumor tissue from edematous, necrotic, or fibrotic tissue, leading to discrepancies between the real tumor size and signal abnormalities in MRI (8). In a mixed group of patients including both high- and low-grade gliomas, MRI yielded a sensitivity (i.e., proportion of individuals who had the disease who tested positive) of over $90 \%$ for the detection of tumor tissue, but specificity (i.e., proportion of individuals without the disease who tested negative) was only 53\% (9).

For more than $20 \mathrm{y}$ the amino acid tracer ${ }^{11} \mathrm{C}$-methionine has been used in the management of gliomas (10). In several studies, the tracer was investigated with regard to various indications such as diagnosis, grading, planning of biopsies, planning of surgery, postoperative revaluation, and planning of radiation $(11,12)$. More recently, the amino acid tracer ${ }^{18} \mathrm{~F}$ fluoroethyltyrosine was introduced for similar indications (8).

Moreover, studies have indicated that PET using radiolabeled amino acids is superior to CT and MRI in estimating the size and extension of the tumor in high-grade gliomas (8). It has been demonstrated that, compared with MRI alone, the combined use of MRI and amino acid PET distinguishes more accurately between tumor tissue and peritumoral unaffected brain tissue (9).

Accordingly, the current guidelines of the Neuroimaging Committee of the European Association of Nuclear Medicine and the German Society for Nuclear Medicine recommend the use of amino acid PET for therapy planning of the surgical resection of gliomas $(13,14)$.

To date, the reimbursement of PET in the management of primary brain tumors varies among different countries. In most countries, the reimbursement is restricted to the use of ${ }^{18} \mathrm{~F}-\mathrm{FDG}(15,16)$. In Germany, the use of PET is refunded for neither ${ }^{18}$ F-FDG nor amino acid tracers ((17); www.g-ba.de). Reimbursements for in-patient use of PET are included in the lump compensation (German Diagnosis Related Groups, www.g-drg.de). 
In 2006, the German Federal Joint Committee assigned the Institute for Quality and Efficiency in Healthcare (IQWiG) to evaluate the diagnostic validity of PET for the assessment of recurrent high-grade gliomas after initial treatment (www. iqwig.de [D06-01D]). According to the institute's evaluation, there was only insufficient evidence for PET to improve the diagnosis or prognosis of recurrent gliomas. In contrast to the IQWiG report, this paper analyzes the use of PET at the initial diagnosis of gliomas. In a recent study, Pirotte et al. (18) have shown that the use of ${ }^{11} \mathrm{C}$-methionine PET-guided surgery may increase the mean survival of patients with supratentorial high-grade gliomas (World Health Organization grades III and IV). These results indicate for the first time, to our knowledge, that the use of PET-guided surgery may increase survival of the patients.

To date, there is no economic analysis evaluating the use of PET in the management of gliomas. The aim of this analysis was to estimate the cost-effectiveness of amino acid PET, compared with MRI alone, in gliomas for guiding the initial surgical resection of the tumor.

\section{MATERIALS AND METHODS}

To account for the cost-effectiveness of amino acid PET and MRI, compared with MRI alone, a decision tree using TreeAge Pro 2009 (TreeAge, Inc.) was constructed (Fig. 1; Table 2). The analysis was performed from the perspective of the statutory health insurance in Germany.

\section{Decision-Tree Model}

To evaluate the cost-effectiveness of ${ }^{11} \mathrm{C}$-methionine PET in guiding the resection of high-grade gliomas, the combined use of MRI and PET was compared with MRI alone (no PET). The outcome was defined as the mean survival of the patients in years.

The decision-tree model begins at the point where the diagnosis of a high-grade glioma is considered as highly likely, resulting in the decision for surgical resection.

In this model, we use operability as a theoretic construct. It is defined according to tumor volume and extension in the PET scan. However, it does not imply that a PET scan has to be performed. Hence, the no-PET strategy has the same probability of complete or incomplete operability even if no PET scan is obtained. In our model, this concept is used to illustrate the group of patients that would not benefit from a PET scan because a complete resection of the tumor is impossible.

The probabilities for the first-chance node (complete operability and incomplete operability) depend on the tumor volume defined by the PET scan. Although complete operability implies the ability to remove the whole-tumor volume, incomplete operability does not allow complete resection because of different reasons (e.g., a total resection may endanger vitally important parts of the brain).

The second-chance node describes the impact of the PET scan on the surgical performance. The surgery may result in a complete resection of PET-positive tumor (i.e., no residual tumor on postoperative PET scan) or in an incomplete resection of the tumor.

The upper branches show the options to perform PET to guide the surgical resection of the cancer. Given a complete operability, we assumed that the use of PET would lead to a negative postoperative PET scan result. Therefore, we attributed a probability of 1 to complete resection of PET-positive tumor.

Time of survival in the case of complete or incomplete resection was based on published data (18). Pirotte et al. found that a patient with a complete resection had a mean survival of

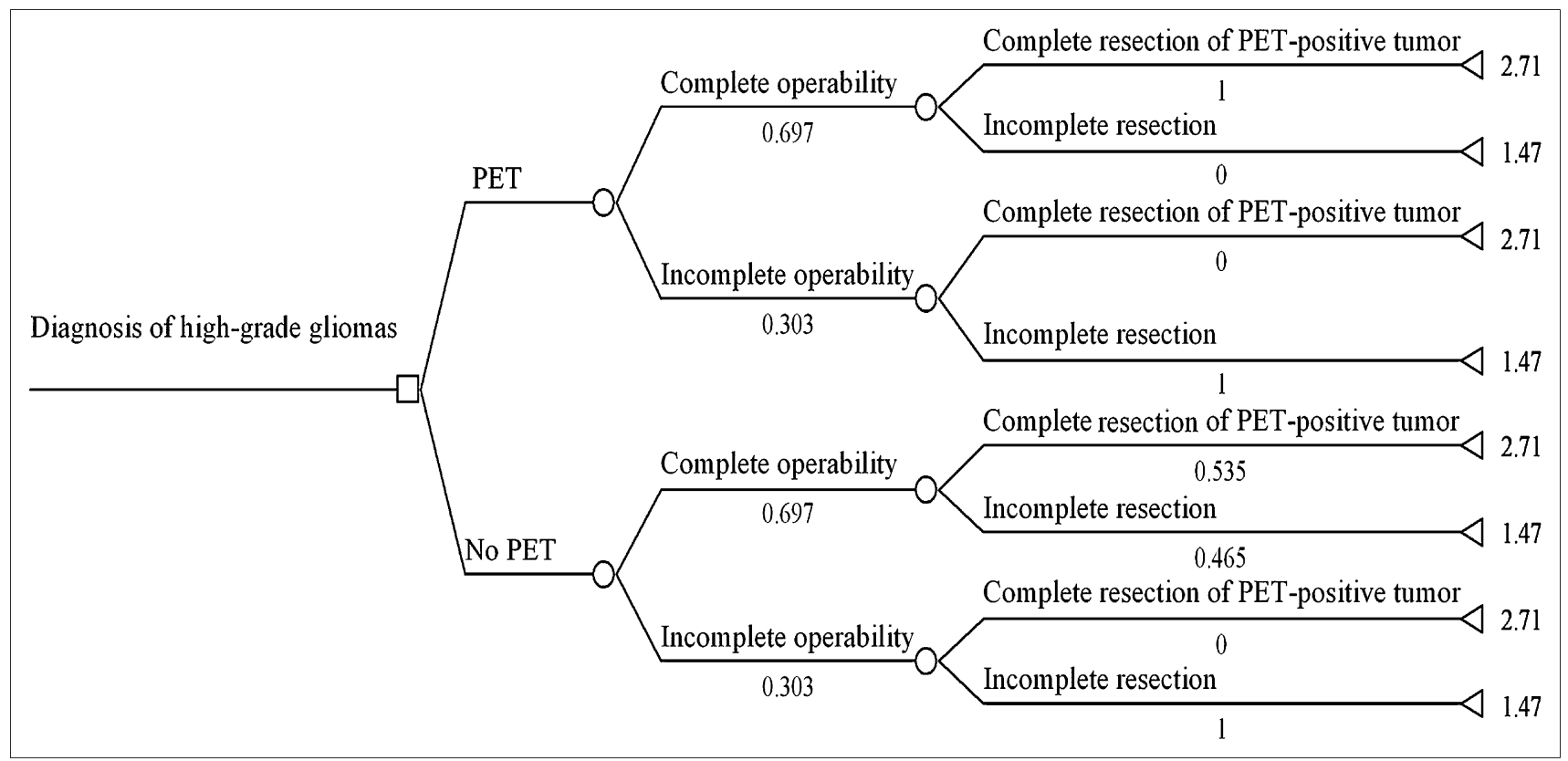

FIGURE 1. Decision-tree-model effectiveness of ${ }^{11} \mathrm{C}$-methionine PET in therapy planning of surgery for patients with high-grade gliomas. Model includes 2 alternative strategies of use of MRI alone (no PET) to guide resection of high-grade gliomas and of combined use of MRI and PET (PET). Mean survival of patients (years) is defined as outcome. Decision-tree model begins at point at which decision for surgical resection is made and MRI scan of brain has already been acquired. $\mathrm{O}=$ Chance node; $\boldsymbol{\Psi}=$ termination node. 
32.5 mo, whereas a patient with an incomplete resection had a significantly shorter mean survival of 17.6 mo $(P<0.001)$. These values were applied as possible outcomes.

For the no-PET strategy, the completeness of the resection depended on MRI alone. To analyze the results of the no-PET strategy, Pirotte et al. (18) retrospectively compared biopsies of tumor volumes defined by MRI with those defined by PET. These probabilities were applied in our model. The tumor volume on the MRI scan might be defined as equal to or larger than that on the PET scan, resulting in a complete resection (i.e., no residual tumor on the postoperative PET scan). Therefore, in these cases we applied the same outcome as for complete resection in the PET strategy. A PET scan would not have been of additional benefit for the patient.

Alternatively, the MRI scan might define the tumor volume smaller than would the PET scan, resulting in an incomplete tumor resection. In these cases, the proportion of incomplete resection in the no-PET branch was calculated as the number of the PETpositive areas outside the MRI-positive volume (18).

We attributed the same outcome as for residual cancer in the postoperative PET scan. In these cases, the patient would benefit from the PET scan because it would lead to a complete tumor resection.

Incomplete operability results in an incomplete resection of the tumor irrespective of the applied strategy. Therefore, for both strategies at the second-chance node after alternative incomplete operability, we attributed a probability of 1 for incomplete resection.

The cost-effectiveness of ${ }^{11} \mathrm{C}$-methionine PET was then calculated using the incremental cost-effectiveness ratio (ICER) (19):

$$
\mathrm{ICER}=\frac{([\text { costs MRI }+ \text { PET }]-[\text { costs MRI }])}{([\text { life years gained MRI }+ \text { PET }]-[\text { life years gained MRI }])}
$$

\section{Calculation of Costs}

Because the costs of amino acid PET are currently not covered by the German statutory health insurance, they were obtained from the scale for medical procedures not accounted for by the German national health insurance system (http://www.e-bis.de/goae/ defaultFrame.htm). Although the baseline charge rate (einfacher Gebührensatz) is calculated by multiplying this score by $€ 0.058$ ( $€ 1 \sim$ U.S.\$1.3), for an additional scenario a severity-based multiplying factor was used. This factor is referred to as admissiblecharge rate (zulässiger Gebührensatz) and enables the calculation of costs according to various factors such as the difficulty of the procedure or the qualification of the executing person (Table 1).

In addition to the costs of amino acid PET, the costs of the tracer, including the value-added tax (VAT) of $19 \%$, have to be considered. Many university hospitals applying amino acid PET have the capacity (i.e., cyclotron) to produce the tracers themselves. Because of the short physical half-life of the radiotracer, ${ }^{11} \mathrm{C}$-methionine is not commercially available. In contrast, ${ }^{18} \mathrm{~F}$-fluoroethyltyrosine is an ${ }^{18} \mathrm{~F}$-labeled amino acid that can be produced in large amounts and is transportable over long distances. In Germany, ${ }^{18} \mathrm{~F}$-fluoroethyltyrosine is available via commercial enterprises. To represent the range of costs, we calculated the mean of the price for the tracer of 2 different enterprises and 1 in-house production (€616).

Additional costs due to side effects were not included because significant negative side effects caused by PET are highly unlikely $(9,20)$. Indirect costs are not considered because they are not relevant from the perspective of health insurance.

\section{Sensitivity Analyses}

To test for the robustness of the results, we calculated deterministic and probabilistic sensitivity analyses. Because of a lack of data, intervals for the sensitivity analysis were based on expert opinion (Table 2).

Moreover, we performed probabilistic sensitivity analyses using Monte Carlo simulation (21). We chose $\gamma$-distributions for costs (22), exponential distributions for survival time, and triangular distributions for probabilities $(23,24)$. On the basis of the initial definition of the intervals by the expert, we fitted the respective distributions for all variables (Table 2). Afterward, the expert was questioned again to ensure the plausibility of the distributions. On the basis of these distributions, we performed a second-order Monte Carlo simulation with 10,000 samples.

\section{RESULTS}

Compared with MRI alone, the combined use of PET and MRI resulted in an ICER of $€ 1,185 / 2.334-1.932 \mathrm{y}=€ 2,948$ per life-year gained for the baseline scenario and an ICER of $€ 1,650 / 2.334-1.932 \mathrm{y}=€ 4,105$ per life-year gained for the admissible-charge-rate scenario (Table 3).

\section{1-Way Sensitivity Analyses}

The 1-way sensitivity analyses revealed that only the survival time would alter the decision between the 2 alternative strategies. For all other parameters, the combined use of PET and MRI is preferred for all values within the defined ranges (Supplemental Table 1; supplemental materials are available online only at http://jnm.snmjournals.org).

\section{Probabilistic Sensitivity Analyses}

The statistics of the Monte Carlo simulations are presented in Table 3 . The respective incremental cost-effectiveness scatterplots are demonstrated in Figure 2 for the

\section{TABLE 1}

Costs

\begin{tabular}{lcr}
\hline \multicolumn{1}{c}{ Description } & Admissible-charge rate & Baseline \\
\hline Detailed consultation (3) & $€ 20.10$ & $€ 8.74$ \\
Report on diagnostic findings (75) & $€ 17.43$ & $€ 7.58$ \\
Intravenous injection (253) & $€ 9.38$ & $€ 4.08$ \\
Whole-body tumor scintigraphy (5,431) & $€ 236.07$ & $€ 131.15$ \\
PET with quantitative analysis (5,489) & $€ 786.89$ & $€ 417.15$ \\
Radioactive tracer plus 19\% value-added tax & $€ 616$ & $€ 616$ \\
Sum (rounded) & $€ 1,686$ & $€ 1,185$
\end{tabular}

Numbers in parentheses refer to ciphers of German cost scale for medical procedures. 
TABLE 2

Parameters Used for Decision-Tree Analysis

\begin{tabular}{|c|c|c|c|}
\hline Parameter & $\begin{array}{c}\text { Value applied in } \\
\text { decision-tree } \\
\text { model }\end{array}$ & $\begin{array}{l}\text { Plausible } \\
\text { range }\end{array}$ & Distribution \\
\hline Probability of complete operability & 0.697 & $0.4-0.8$ & Triangular (minimum, 0.4; likeliest, 0.65; maximum, 0.8) \\
\hline $\begin{array}{l}\text { Probability of complete resection without } \\
\text { PET }\end{array}$ & 0.68 & $0.26-0.8$ & Triangular (minimum, 0.26; likeliest, 0.6; maximum, 0.8) \\
\hline Costs (baseline $[€]$ ) & 1,185 & $829-1,541$ & $\gamma$ (mean, 1,185; SD, 181.63) \\
\hline Costs (admissible-charge rate [€]) & 1,686 & $1,180-2,192$ & $\gamma$ (mean, 1,686; SD, 258.16) \\
\hline Survival with PET (y) & 2.71 & $1.5-3.0$ & $\exp (\lambda, 0.369)$ \\
\hline Survival without PET (y) & 1.47 & $0.5-1.8$ & $\exp (\lambda, 0.68)$ \\
\hline
\end{tabular}

Parameters are used to calculate baseline scenario, including ranges and distributions for sensitivity analyses (variables refer to Fig. 1 and parameters in first column of table).

baseline scenario and in Figure 3 for the admissible-chargerate scenario.

In the baseline scenario, in $61 \%$ of the samples PET was superior to no PET, given a willingness to pay of $€ 30,000 / y$. In $35 \%$ of the iterations, PET was inferior independently of a threshold, and in $4 \%$ PET was inferior with regard to the actual willingness to pay.

In the admissible-charge-rate scenario, in $60 \%$ of the samples PET was superior to no PET (given the willingness-to-pay threshold), in 35\% PET was inferior (independently of a threshold), and in 5\% the alternative PET was inferior with regard to the actual willingness to pay.

\section{DISCUSSION}

On basis of the results of this decision-tree analysis, the combined use of MRI and PET appears to be superior to the use of MRI alone in the surgical therapy planning of supratentorial high-grade gliomas. Compared with MRI alone, the ICER of PET and MRI was less than $€ 5,000$ per life-year gained, even if the admissible-charge-rate scenario was assumed, indicating that PET in addition to MRI may be a cost-effective option.
This is the first, to our knowledge, cost-effectiveness study of amino acid PET-guided surgery for high-grade gliomas. Our analysis has 2 major strengths: first, because various factors may affect the costs, particularly the costs for the tracer substances, 2 scenarios (baseline and admissible-charge rate) were calculated. Second, the costs of PET used for this analysis were based on a reimbursement scale for medical procedures resulting in robust assumptions for the application of this add-on technology. Additional costs due to technologic difficulties or training of care givers are included in the admissible-charge-rate scenario. For calculating the costs of the tracer, 2 options (in-house production of the tracer and acquiring it by purchase) were considered, resulting in a realistic representation of the economic consequences of PET.

The calculations of costs of PET are well in line with those of other studies. For example, in studies from the United States, costs ranged from $\$ 1,200$ to $\$ 2,200$ (corresponding currently to about $€ 880$ to $€ 1,600(25,26))$, and a study from France applied costs of $€ 1,034$ (27). Although in these studies costs are related to PET with ${ }^{18}$ F-FDG (the most commonly used PET tracer) and no study has evalu-

TABLE 3

Statistics Resulting from Monte Carlo Simulations

\begin{tabular}{|c|c|c|c|c|c|c|}
\hline \multirow[b]{2}{*}{ Statistic } & \multicolumn{3}{|c|}{ Baseline scenario } & \multicolumn{3}{|c|}{ Admissible-charge-rate scenario } \\
\hline & $\begin{array}{l}\text { Costs } \\
\text { (PET) }\end{array}$ & $\begin{array}{c}\text { Life-years } \\
\text { gained, PET }\end{array}$ & $\begin{array}{c}\text { Life-years } \\
\text { gained, no PET }\end{array}$ & $\begin{array}{l}\text { Costs } \\
\text { (PET) }\end{array}$ & $\begin{array}{c}\text { Life-years } \\
\text { gained, PET }\end{array}$ & $\begin{array}{c}\text { Life-years } \\
\text { gained, no PET }\end{array}$ \\
\hline Mean & 1,686 & 2.27 & 1.938 & 1,186 & 2.237 & 1.915 \\
\hline SD & 257 & 1.853 & 1.417 & 180 & 1.787 & 1.384 \\
\hline Minimum & 934 & 0.006 & 0.007 & 641 & 0.015 & 0.012 \\
\hline $2.50 \%$ & 1,221 & 0.236 & 0.227 & 862 & 0.233 & 0.229 \\
\hline $10 \%$ & 1,364 & 0.522 & 0.498 & 962 & 0.518 & 0.499 \\
\hline Median & 1,672 & 1.766 & 1.603 & 1,177 & 1.756 & 1.582 \\
\hline $90 \%$ & 2,026 & 4.652 & 3.831 & 1,428 & 4.550 & 3.793 \\
\hline $97.50 \%$ & 2,220 & 7.114 & 5.586 & 1,562 & 6.971 & 5.484 \\
\hline Maximum & 2,785 & 20.002 & 12.005 & 2,019 & 16.021 & 10.933 \\
\hline
\end{tabular}




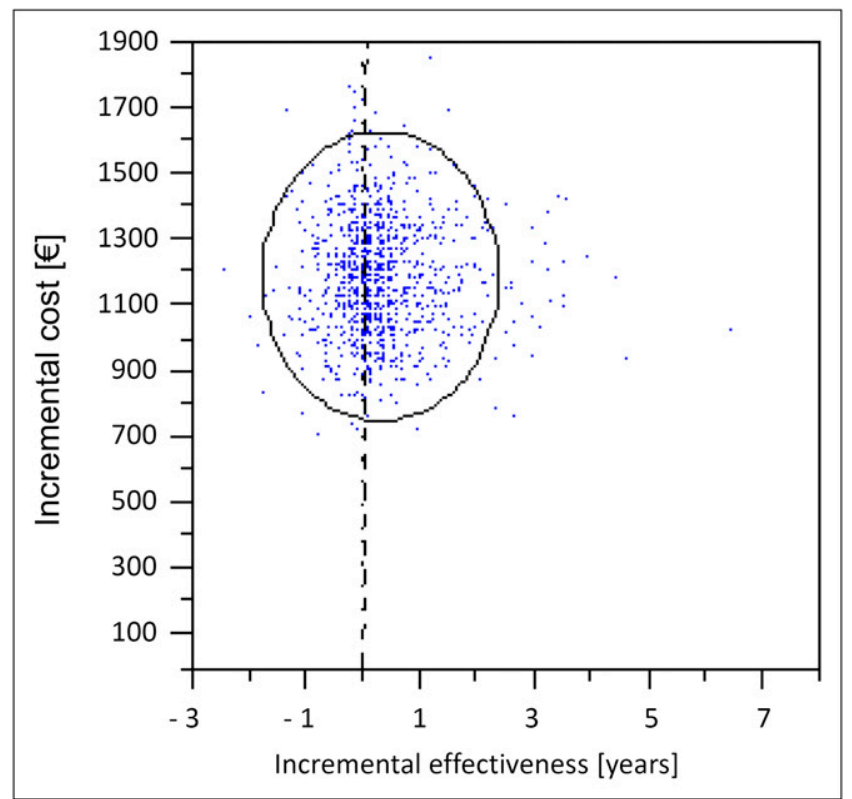

FIGURE 2. Distribution of results from Monte Carlo simulation (blue dots) with regard to incremental cost-effectiveness of PET vs. no PET for baseline scenario. Within circle are $95 \%$ of values. Dotted horizontal line marks incremental costs of $€ 0$. Dotted vertical line depicts incremental effectiveness of 0 . Dashed line (almost overlying dotted line) represents willingness to pay, which was set to $€ 30,000$ per year.

ated the calculation of costs of amino acid PET so far, we would not expect remarkable differences.

The decision-tree model is based on the assumption that a complete resection of the tumor leads to a more favorable prognosis for the patients. This assumption is in accordance with several studies (28-31) and with international guidelines that recommend the maximal safe resection for newly diagnosed supratentorial malignant glioma in adults (level II recommendation indicating a moderate degree of clinical certainty (32)).

Recently, 5-aminolevulinic acid (5-ALA), a metabolic marker of glioma cells, is increasingly used to identify tumor tissue intraoperatively. Compared with conventional surgery, the completeness of surgical resection can be improved using 5-ALA, resulting in an improved progression-free survival (30). A recent study, however, has shown that 5-ALA fluorescence, compared with amino acid PET, has a limited sensitivity to detect tumor tissue, especially in low-grade gliomas (33). Future studies may directly compare the results of resection guided either by 5-ALA or by amino acid PET.

For this analysis, several limitations have to be considered. First, only limited clinical evidence is available to build the model because the data could be derived only from a nonrandomized controlled study. The study of Pirotte et al. (18), from which clinical data were taken, did not have a control group based on the results of MRI alone. To evaluate the diagnostic methods, the results of MRI alone and MRI combined with PET were analyzed separately after the surgical resection. Moreover, in this study the physicians were not masked. Thus, an expectation bias (i.e., the tendency of the physicians to evaluate data according to their expectations (34)) might have influenced the results.

It may be argued that an evaluation of the cost-effectiveness of PET-guided resection of high-grade gliomas presupposes efficacy data based on randomized controlled trials. However, this would require withholding the PET scan from patients, resulting in a decreased mean survival. Even if the underlying evidence can only be considered preliminary, some authors judge the withholding of PET as inappropriate from an ethical point of view $(35,36)$.

Although Pirotte et al. (18) addressed for the first time the influence of PET-guided resection of brain tumors on survival of the patients, the superiority of amino acid PET, compared with MRI, in the definition of the tumor extent has been repeatedly demonstrated $(8,9,12,37,38)$. In addition, a recent study on the use of amino acid PET after radiochemotherapy indirectly confirmed the results of Pirotte et al. (18). They demonstrated that patients with an early decrease in posttherapeutic PET uptake had both an increased median disease-free survival ( 10.3 vs. $5.8 \mathrm{mo} ; P<0.01)$ and an increased overall survival (not reached vs. $9.3 \mathrm{mo} ; P<$ 0.001), compared with patients with higher uptakes in posttherapeutic PET (39). Similar results have been found in studies with amino acid SPECT tracers, which are comparable to the amino acid PET tracers (40). Although none of these results was based on randomized controlled trials, this is the best available evidence.

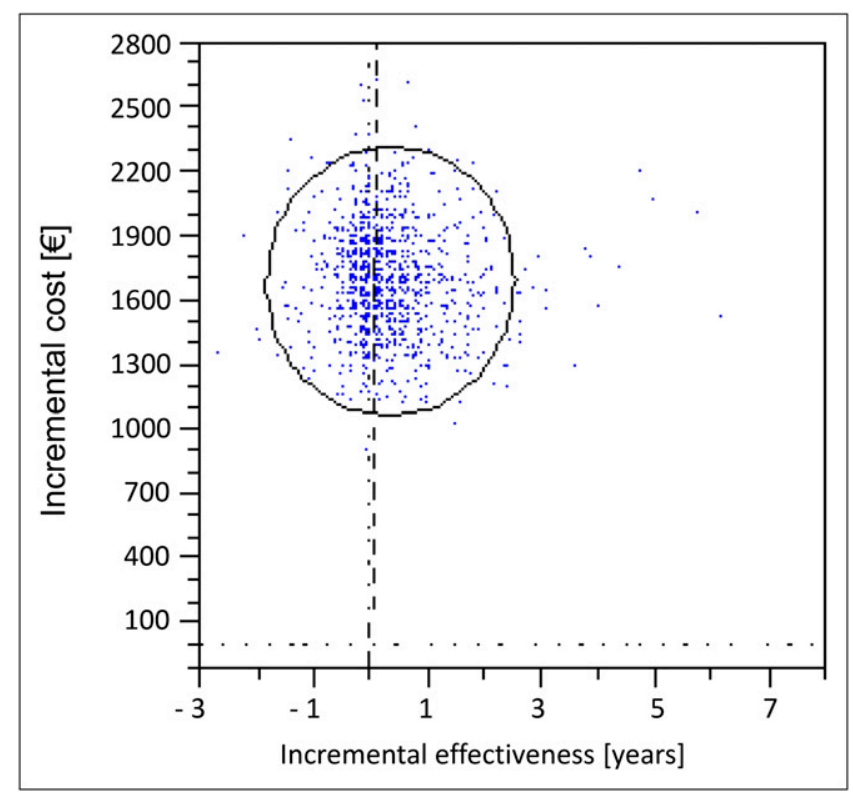

FIGURE 3. Distribution of results from Monte Carlo simulation (blue dots) with regard to incremental cost-effectiveness of PET vs. no PET for admissible-charge-rate scenario. Within circle are $95 \%$ of values. Dotted horizontal line marks incremental costs of $€ 0$. Dotted vertical line depicts incremental effectiveness of 0 . Dashed line (almost overlying dotted line) represents willingness to pay, which was set to $€ 30,000$ per year. 
Second, the model does not consider adverse events due to false-positive PET scan results. A false-positive PET scan finding would result in an unnecessary excision of healthy tissue. This issue was not addressed by the model because there were no empiric data.

Third, because of a lack of data the model does not allow the consideration of a potential reduction of the removed tumor volume as a result of PET. Because the delineation with MRI alone may overestimate the tumor volume, the additional information from the PET scan may lead to a decrease of removed tissue, possibly resulting in reduced morbidity and improved quality of life for patients.

Fourth, PET is considered the gold standard to verify the completeness of surgical resection, leading to a sensitivity and specificity of 1 for the PET scan, which might be an overestimation. However, studies have demonstrated a high sensitivity and specificity of $90 \%$ for ${ }^{11} \mathrm{C}$-methionine PET to detect tumor tissue and a better ability to detect tumor tissue than other diagnostic means such as CT or MRI (12). Additionally, in a recent study on gliomas in children, postoperative ${ }^{11} \mathrm{C}$-methionine PET presented as neither falsepositive nor false-negative and was significantly better than MRI $(P<0.05)(41)$.

Moreover, evidence of diagnostic validity is difficult to establish because it would imply the availability of histologic specimens or surgical samples of brain areas that are negative in the PET scan. For ethical reasons it is difficult to justify biopsies or resection of brain tissue that is probably healthy.

Furthermore, in the decision-tree model we assumed a likelihood of 1 for the completeness of the resection using PET. Obviously, this is an unrealistic scenario because intraoperative problems, particularly for technical reasons, might occur. However, we did not consider these problems because of a lack of data on the likelihood for the completeness of resection using PET and because intraoperative problems were not considered for both alternatives (i.e., MRI vs. MRI and PET). Therefore, the calculation of the ICER was not affected.

Finally, our decision-tree model neglects possible patientrelated benefits in terms of morbidity. For example, if the PET scan indicates inoperability of the tumor the patient will also benefit because possible surgery-related morbidity is avoided. Studies reported serious postoperative morbidity varying between $8 \%$ and $20 \%(42,43)$. Thus, if aspects of morbidity had been additionally considered, PET would have been even more cost-effective.

The economic impact of the use of amino acid PET in patients with gliomas depends on the total costs of diagnosis and treatment. A recent review reported that the ICER of the use of temozolomide, a chemotherapeutic treatment option, was between $€ 37,361$ per life-year gained and $€ 42,912$ per quality-adjusted life-year gained. The treatment costs for temozolomide were between $€ 27,365$ and $€ 39,092$ and amounted to approximately $40 \%$ of the total treatment costs (44), demonstrating that the use of amino acid PET will have a relatively low impact on total treatment costs of patients with gliomas but may have a strong impact on treatment efficiency. Studies have demonstrated that a complete resection of a malignant glioma improves survival. Moreover, if, in addition to the surgery, medical therapies are applied, their performance increases with decreasing tumor tissue. For example, it was observed that radiochemotherapy followed by adjuvant chemotherapy was more effective in patients treated by complete resection than in those treated by incomplete resection $(45,46)$.

\section{CONCLUSION}

The results of this analysis indicate that amino acid PET may be a cost-effective tool in the therapy planning of surgical resection of supratentorial high-grade gliomas. Because the model is based on limited empiric evidence, additional studies of high methodologic quality addressing survival time and quality of life are needed to confirm the results.

\section{DISCLOSURE STATEMENT}

The costs of publication of this article were defrayed in part by the payment of page charges. Therefore, and solely to indicate this fact, this article is hereby marked "advertisement" in accordance with 18 USC section 1734.

\section{ACKNOWLEDGMENT}

No potential conflict of interest relevant to this article was reported.

\section{REFERENCES}

1. Osoba D, Aaronson NK, Muller M, et al. Effect of neurological dysfunction on health-related quality of life in patients with high-grade glioma. J Neurooncol. 1997;34:263-278.

2. Stewart LA. Chemotherapy in adult high-grade glioma: a systematic review and meta-analysis of individual patient data from 12 randomised trials. Lancet. 2002;359:1011-1018.

3. DeAngelis LM. Brain tumors. N Engl J Med. 2001;344:114-123.

4. DeAngelis LM. Anaplastic glioma: how to prognosticate outcome and choose a treatment strategy. J Clin Oncol. 2009;27:5861-5862.

5. Stupp R, Mason WP, van den Bent MJ, et al. Radiotherapy plus concomitant and adjuvant temozolomide for glioblastoma. N Engl J Med. 2005;352:987-996.

6. Stupp R, Roila F. Malignant glioma: ESMO clinical recommendations for diagnosis, treatment and follow-up. Ann Oncol. 2008;19(suppl 2):ii83-ii85.

7. Chen W. Clinical applications of PET in brain tumors. J Nucl Med. 2007;48:14681481 .

8. Langen KJ, Hamacher K, Weckesser M, et al. $O$ - $\left(2-\left[{ }^{18} \mathrm{~F}\right]\right.$ fluoroethyl)-L-tyrosine: uptake mechanisms and clinical applications. Nucl Med Biol. 2006;33:287-294.

9. Pauleit D, Floeth F, Hamacher K, et al. $O-\left(2-\left[{ }^{18} \mathrm{~F}\right]\right.$ fluoroethyl $)$-L-tyrosine PET combined with MRI improves the diagnostic assessment of cerebral gliomas. Brain. 2005;128:678-687.

10. Mosskin M, Ericson K, Hindmarsh T, et al. Positron emission tomography compared with magnetic resonance imaging and computed tomography in supratentorial gliomas using multiple stereotactic biopsies as reference. Acta Radiol. 1989;30:225-232.

11. Grosu AL, Weber WA, Riedel E, et al. L-(methyl- $\left.{ }^{11} \mathrm{C}\right)$ methionine positron emission tomography for target delineation in resected high-grade gliomas before radiotherapy. Int J Radiat Oncol Biol Phys. 2005;63:64-74.

12. Singhal T, Narayanan TK, Jain V, Mukherjee J, Mantil J. ${ }^{11} \mathrm{C}-\mathrm{L}-$-methionine positron emission tomography in the clinical management of cerebral gliomas. Mol Imaging Biol. 2008;10:1-18.

13. Langen KJ, Bartenstein $\mathrm{P}$, Boecker $\mathrm{H}$, et al. German guidelines for brain tumour imaging by PET and SPECT using labelled amino acids [in German]. Nuklearmedizin. 2011;50:167-173.

14. Vander Borght T, Asenbaum S, Bartenstein P, et al. EANM procedure guidelines for brain tumor imaging using labelled amino acid analogues. Eur J Nucl Med Mol Imaging. 2006;33:1374-1380. 
15. Dietlein M, Schicha H. Reimbursement of the PET in oncology in Europe: a questionnaire based survey [in German]. Nuklearmedizin. 2003;42:80-85.

16. Buck AK, Herrmann K, Stargardt T, Dechow T, Krause BJ, Schreyogg J. Economic evaluation of PET and PET/CT in oncology: evidence and methodologic approaches. J Nucl Med Technol. 2010;38:6-17.

17. Federal Joint Committee Bekanntmachungen. Bundesanzeiger. 2006;43:1374.

18. Pirotte BJ, Levivier M, Goldman S, et al. Positron emission tomography-guided volumetric resection of supratentorial high-grade gliomas: a survival analysis in 66 consecutive patients. Neurosurgery. 2009;64:471-481.

19. Karlsson G, Johannesson M. The decision rules of cost-effectiveness analysis. Pharmacoeconomics. 1996;9:113-120.

20. Pauleit D, Floeth F, Herzog H, et al. Whole-body distribution and dosimetry of O-(2-[ ${ }^{18}$ F]fluoroethyl)-L-tyrosine. Eur J Nucl Med Mol Imaging. 2003;30:519524.

21. Doubilet P, Begg CB, Weinstein MC, Braun P, McNeil BJ. Probabilistic sensitivity analysis using Monte Carlo simulation: a practical approach. Med Decis Making. 1985;5:157-177.

22. Benedict A, Figlin RA, Sandstrom P, et al. Economic evaluation of new targeted therapies for the first-line treatment of patients with metastatic renal cell carcinoma. BJU Int. 2011;108:665-672.

23. Meltzer MI, Dennis DT, Orloski KA. The cost effectiveness of vaccinating against Lyme disease. Emerg Infect Dis. 1999;5:321-328.

24. Burgos JL, Gaebler JA, Strathdee SA, Lozada R, Staines H, Patterson TL. Costeffectiveness of an intervention to reduce HIV/STI incidence and promote condom use among female sex workers in the Mexico-US border region. PLOS ONE. 2010;5: e11413.

25. Scott WJ, Shepherd J, Gambhir SS. Cost-effectiveness of FDG-PET for staging non-small cell lung cancer: a decision analysis. Ann Thorac Surg. 1998;66:18761883.

26. Valk PE, Pounds TR, Tesar RD, Hopkins DM, Haseman MK. Cost-effectiveness of PET imaging in clinical oncology. Nucl Med Biol. 1996;23:737-743.

27. Lejeune $\mathrm{C}$, Bismuth MJ, Conroy $\mathrm{T}$, et al. Use of a decision analysis model to assess the cost-effectiveness of ${ }^{18} \mathrm{~F}$-FDG PET in the management of metachronous liver metastases of colorectal cancer. J Nucl Med. 2005;46:2020-2028.

28. Ammirati M, Vick N, Liao YL, Ciric I, Mikhael M. Effect of the extent of surgical resection on survival and quality of life in patients with supratentorial glioblastomas and anaplastic astrocytomas. Neurosurgery. 1987;21:201-206.

29. Simpson JR, Horton J, Scott C, et al. Influence of location and extent of surgical resection on survival of patients with glioblastoma multiforme: results of three consecutive Radiation Therapy Oncology Group (RTOG) clinical trials. Int J Radiat Oncol Biol Phys. 1993;26:239-244.

30. Stummer W, Pichlmeier U, Meinel T, Wiestler OD, Zanella F, Reulen HJ. Fluorescence-guided surgery with 5-aminolevulinic acid for resection of malignant glioma: a randomised controlled multicentre phase III trial. Lancet Oncol. 2006;7:392-401.
31. Vuorinen V, Hinkka S, Farkkila M, Jaaskelainen J. Debulking or biopsy of malignant glioma in elderly people - a randomised study. Acta Neurochir (Wien). 2003;145:5-10.

32. Olson JJ, Fadul CE, Brat DJ, Mukundan S, Ryken TC. Management of newly diagnosed glioblastoma: guidelines development, value and application. J Neurooncol. 2009;93:1-23.

33. Floeth FW, Sabel M, Ewelt C, et al. Comparison of ${ }^{18}$ F-FET PET and 5-ALA fluorescence in cerebral gliomas. Eur J Nucl Med Mol Imaging. 2011;38:731-741.

34. Jeng M. A selected history of expectation bias in physics. Am J Phys. 2006;74:578583

35. Temple R, Ellenberg SS. Placebo-controlled trials and active-control trials in the evaluation of new treatments: part 1-ethical and scientific issues. Ann Intern Med. 2000;133:455-463.

36. Miller FG, Brody H. What makes placebo-controlled trials unethical? Am J Bioeth. 2002;2:3-9.

37. Kracht LW, Miletic H, Busch S, et al. Delineation of brain tumor extent with

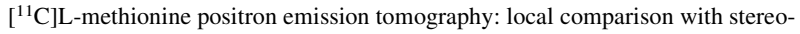
tactic histopathology. Clin Cancer Res. 2004;10:7163-7170.

38. Miwa K, Shinoda J, Yano H, et al. Discrepancy between lesion distributions on methionine PET and MR images in patients with glioblastoma multiforme: insight from a PET and MR fusion image study. J Neurol Neurosurg Psychiatry. 2004;75:14571462.

39. Piroth MD, Pinkawa M, Holy R, et al. Prognostic value of early $\left[{ }^{18} \mathrm{~F}\right]$ Fluoroethyltyrosine positron emission tomography after radiochemotherapy in glioblastoma multiforme. Int J Radiat Oncol Biol Phys. 2011;80:176-184.

40. Weber WA, Dick S, Reidl G, et al. Correlation between postoperative 3-[ $\left.{ }^{123} \mathrm{I}\right]$ iodo-L-alpha-methyltyrosine uptake and survival in patients with gliomas. $\mathrm{J} \mathrm{Nucl}$ Med. 2001;42:1144-1150.

41. Pirotte BJ, Lubansu A, Massager N, et al. Clinical impact of integrating positron emission tomography during surgery in 85 children with brain tumors. J Neurosurg Pediatr. 2010;5:486-499.

42. Fadul C, Wood J, Thaler H, Galicich J, Patterson RH Jr, Posner JB. Morbidity and mortality of craniotomy for excision of supratentorial gliomas. Neurology. 1988;38:1374-1379.

43. Salcman M, Scholtz H, Kaplan RS, Kulik S. Long-term survival in patients with malignant astrocytoma. Neurosurgery. 1994;34:213-219.

44. Uyl-de Groot CA, Stupp R. van der BM. Cost-effectiveness of temozolomide for the treatment of newly diagnosed glioblastoma multiforme. Expert Rev Pharmacoecon Outcomes Res. 2009;9:235-241.

45. Piroth MD, Gagel B, Pinkawa M, Stanzel S, Asadpour B, Eble MJ. Postoperative radiotherapy of glioblastoma multiforme: analysis and critical assessment of different treatment strategies and predictive factors. Strahlenther Onkol. 2007; 183:695-702.

46. Stummer W, Tonn JC, Mehdorn HM, et al. Counterbalancing risks and gains from extended resections in malignant glioma surgery: a supplemental analysis from the randomized 5-aminolevulinic acid glioma resection study. J Neurosurg. 2011; 114:613-623. 